\title{
Dietary scores at midlife and healthy ageing in a French prospective cohort
}

\author{
Karen E. Assmann ${ }^{1}$, Valentina A. Andreeva ${ }^{1}$, Géraldine M. Camilleri ${ }^{1}$, Eric O. Verger ${ }^{2,3}$, Claude Jeandel $^{4}$, \\ Serge Hercberg ${ }^{1,5}$, Pilar Galan ${ }^{1}$ and Emmanuelle Kesse-Guyot ${ }^{1 *}$ \\ ${ }^{1}$ Equipe de Recherche en Epidémiologie Nutritionnelle (EREN), Centre d'Epidémiologie et Statistiques Sorbonne Paris Cité, \\ Inserm (U1153), Inra (U1125), Cnam, COMUE Sorbonne Paris Cité, Université Paris 13, F-93017 Bobigny, France \\ ${ }^{2}$ Département Nutrition, Institut de Cardiométabolisme et Nutrition (ICAN), Assistance Publique-Hôpitaux de Paris, \\ Pitié-Salpêtrière Hôpital, F-75013 Paris, France \\ ${ }^{3}$ INSERM, UMR_S U1166, Equipe Nutriomique: Nutrition et Obésité; Approches Systémiques (NutriOmics), F-75013 Paris, France \\ ${ }^{4}$ Département de Gériatrie, Centre Balmès, Centre Hospitalier Universitaire (CHU) Montpellier, Université Montpellier I, \\ 34090, Montpellier, France \\ ${ }^{5}$ Département de Santé Publique, Hôpital Avicenne, F-93017 Bobigny, France
}

(Submitted 16 December 2015 - Final revision received 30 March 2016 - Accepted 27 April 2016 - First published online 15 June 2016)

\section{Abstract}

Although nutrition has been advocated as a major determinant of healthy ageing (HA), studies investigating the link between dietary quality and HA are scarce. We investigated the association between adherence to French food-based and nutrient-based guidelines at midlife, as assessed by three dietary scores, and HA. HA was assessed in 2007-2009, among 2329 participants of the SUpplémentation en Vitamines et Minéraux AntioXydants study aged 45-60 years at baseline (1994-1995) and initially free of diabetes, CVD and cancer. HA was defined as not developing any major chronic disease, good physical and cognitive functioning, no limitations in instrumental activities of daily living, no depressive symptoms, no health-related limitations in social life, good overall self-perceived health and no function-limiting pain. Data from repeated 24-h dietary records provided at baseline permitted the computation of the modified French Programme National Nutrition SantéGuideline Score (mPNNS-GS), the Probability of Adequate Nutrient Intake Dietary Score (PANDiet) and the Diet Quality Index-International (DQI-I). Associations of these scores with HA were assessed by logistic regression. In 2007-2009, $42 \%$ of men and $36 \%$ of women met our criteria of HA. After adjustment for potential confounders, higher scores of the mPNNS-GS (OR quartile $4 v$. quartile $11.44 ; 95 \%$ CI $1 \cdot 10,1 \cdot 87$; $\left.P_{\text {trend }}=0.006\right)$ and the PANDiet $\left(1.28 ; 95 \%\right.$ CI 1.00, 1.64; $\left.P_{\text {trend }}=0.03\right)$ were associated with higher odds of HA. We observed no association between DQI-I and HA. In conclusion, this study suggests a beneficial long-term role of high adherence to both food-based and nutrient-based French dietary guidelines for a HA process.

Key words: Nutritional recommendations: Dietary scores: Healthy ageing: Midlife exposures

Over the last few decades, new multidimensional concepts referred to as 'successful ageing' or 'healthy ageing' (HA) have emerged $^{(1)}$. These concepts aim to capture health during ageing as a whole, beyond specific medical conditions or body functions. A large part of these constructs is based on the model proposed by Rowe \& Kahn ${ }^{(2)}$, which defines successful ageing as being at low risk of disease or disability, while maintaining high levels of cognitive and physical functioning, and an active engagement with life. Yet, a multitude of different models has been developed that differ in the choice of the included components $^{(1)}$ as well as in the indicators used to measure these components.
One of the modifiable environmental factors that have been advocated to have a decisive role for HA is diet ${ }^{(3)}$. In France, the official nutritional guidelines for the general public were developed in the context of the national public health nutrition programme (Programme National Nutrition Santé, PNNS) ${ }^{(4)}$. These guidelines include eight food-based items, in order to provide easily understandable public health messages. Moreover, the guidelines include a ninth item, which advises the population to have a regular physical activity, equivalent to at least $30 \mathrm{~min}$ of rapid walking per day. Nutrient-specific reference values such as the French Apports Nutritionnels Conseillés are less comprehensible for the general population, but equally important for

Abbreviations: DQI-I, Diet Quality Index-International; HA, healthy ageing; mPNNS-GS, modified Programme National Nutrition Santé-Guideline Score; PANDiet, Probability of Adequate Nutrient Intake Dietary Score; PNNS-GS, Programme National Nutrition Santé-Guideline Score; SU.VI.MAX, SUpplémentation en Vitamines et Minéraux AntioXydants.

* Corresponding author: K. E. Assmann, fax +33 1483889 31, email k.assmann@eren.smbh.univ-paris13.fr 
prevention research. Although food-based recommendations can also account for non-nutrient components and food matrix effects, they have the disadvantage that the attainment of nutrient reference values is not guaranteed by construction. Thus, food-based and nutrient-based reference systems are complementary approaches whose comparison is of interest.

In epidemiological research, adherence to nutritional recommendations is evaluated by a priori-defined dietary scores ${ }^{(5)}$. Moreover, a posteriori methods are applied to characterise empirically derived overall dietary patterns. To the best of our knowledge, only four cohort studies ${ }^{(6-9)}$ have, to this day, evaluated measures of the overall diet with respect to multidimensional concepts of HA; three studies have identified positive and/or negative roles of specific empirically derived a posteriori dietary patterns ${ }^{(6,7)}$ and a positive role of adherence to the Mediterranean diet ${ }^{(8)}$. Moreover, although higher scores on the Alternative Healthy Eating Index-2010 (AHEI-2010) were positively related to HA in one study ${ }^{(8)}$, scores on the original AHEI (published in 2002) were unrelated to HA in another investigation $^{(6)}$. A further study has reported that higher adherence to the Australian dietary guidelines was associated with a higher probability for $\mathrm{HA}^{(9)}$.

Hence, only one study has directly examined adherence to national nutrition recommendations (i.e. the Australian dietary guidelines), and no study has yet investigated the role of both food-based national dietary guidelines and nutrient reference values with respect to multidimensional concepts of HA

We thus aimed to provide specific data on the pertinence of both food-based dietary guidelines and nutrient reference values in a French context, thus increasing the knowledge on the role of these important elements of public health nutrition strategies for a holistic prevention of age-related health decline. Notably, two different scores reflect adherence to French nutritional recommendations and reference values: the PNNS-Guideline Score (PNNS-GS), reflecting the official food-based guidelines ${ }^{(4)}$, and the Probability of Adequate Nutrient Intake Dietary Score (PANDiet), measuring adequacy to current nutrient reference values $^{(10)}$. Moreover, an international index, the Diet Quality Index-International (DQI-I), comprising both nutrient- and foodgroup items, has been developed to facilitate between-country comparisons ${ }^{(11)}$. This study examined the association between the above-mentioned dietary scores estimated at midlife and HA evaluated 13 years later in a large French cohort.

\section{Methods}

\section{Study design}

Initially, the 'SUpplémentation en Vitamines et Minéraux AntioXydants' study (SU.VI.MAX, 1994-2002) was a French, randomised, double-blind, placebo-controlled, primary, prevention trial with a planned follow-up of 8 years ${ }^{(12)}$. In brief, after a national recruitment campaign with a call for volunteers living in France (women aged 35-60 years, men aged 45-60 years), 12741 subjects returned a completed baseline questionnaire, met the eligibility criteria (no disease likely to hinder active participation or threatened 5-year survival; acceptance of participation constraints; no previous regular supplementation with the tested antioxidants), were present at the inclusion visit, and included into the final study sample. The trial's objective was to investigate a potential effect of antioxidant supplementation at nutritional doses on the incidence of cancers, CVD and mortality ${ }^{(12,13)}$.

The SU.VI.MAX participants were invited, on a voluntary basis, to participate in an additional observational follow-up, the SU.VI.MAX 2 study (2007-2009), 5 years after the end of the trail. This follow-up study included 6860 subjects who completed clinical and neuropsychological examinations and a number of questionnaires ${ }^{(14)}$.

The SU.VI.MAX and SU.VI.MAX 2 studies were conducted according to the guidelines laid down in the Declaration of Helsinki, and all procedures involving human subjects were approved by the Ethics Committee for Studies with Human participants of Paris-Cochin Hospital (CCPPRB nos 706 and 2364, respectively) and the Commission National Informatique et Liberté (CNIL nos 334641 and 907 094, respectively). Written informed consent was obtained from all subjects.

\section{Baseline dietary data (1994-1996)}

During the SU.VI.MAX study, all participants were invited to complete a 24-h dietary record every 2 months, using computerised questionnaires. An instruction manual including validated photographs of $>250$ foods was used to assist participants. Subjects could choose from seven possible portion sizes ${ }^{(15)}$. As previously stated $^{(16)}$, dietary records were considered as invalid if energy intake was $<418.4 \mathrm{~kJ} / \mathrm{d}(<100 \mathrm{kcal} / \mathrm{d})$ or $>25104 \mathrm{~kJ} / \mathrm{d}$ $(>6000 \mathrm{kcal} / \mathrm{d})$. In addition, men reporting $<3347 \mathrm{~kJ} / \mathrm{d}$ $(<800 \mathrm{kcal} / \mathrm{d})$ and women reporting $<2092 \mathrm{~kJ} / \mathrm{d}(<500 \mathrm{kcal} / \mathrm{d})$ across $\geq 1 / 3$ of records were excluded to account for energy under-reporting. Information on alcohol and seafood consumption was obtained by baseline questionnaires, as these food groups tend to be consumed less frequently than others. Alcohol consumption was estimated using a short, validated, semiquantitative dietary questionnaire ${ }^{(17)}$. The computation of food and nutrient intakes was based on all eligible 24-h records collected during the first 2 years following inclusion. On average, $10 \cdot 2$ dietary records (interquartile range: 8-13) were available per participant. The construction of the different dietary scores is presented in Fig. 1 and detailed in the online Supplementary Material S1.

\section{Other baseline (1994-1996) variables}

Data on sex, date of birth, education (primary, secondary, university level), occupational category (homemakers, manual workers, intermediate professions, managerial staff/intellectual profession), living arrangement (living alone, living in a couple), smoking status (never smoked, former or current smoker), physical activity (irregular, $<1 \mathrm{~h}$ of walking/d, $\geq 1 \mathrm{~h}$ of walking/d) and subjective memory complaints (yes/no) were collected using self-administered questionnaires. BMI $\left(\mathrm{kg} / \mathrm{m}^{2}\right)$ was calculated using anthropometric measurements performed by trained personnel. Systolic and diastolic blood pressures were measured three times using a standard mercury sphygmomanometer after lying down for $10 \mathrm{~min}$, and the mean values were calculated. 
PNNS-GS, Estaquio et al. MPNNS-GS: modified score without physical activity component

\begin{tabular}{|c|c|c|c|c|}
\hline \multirow{2}{*}{$\begin{array}{l}\text { Negative } \\
\text { scores } \\
\text { possible }\end{array}$} & $\begin{array}{l}\text { Dietary components } \\
\text { (points attributed) }\end{array}$ & $\begin{array}{l}\text { Physical activity } \\
\text { (points attributed) }\end{array}$ & \multirow{2}{*}{\begin{tabular}{|c|} 
Penalty \\
If energy \\
intake \\
$>105 \%$ of \\
calculated \\
energy \\
needs
\end{tabular}} & $\begin{array}{l}\text { Overall score: } \\
\text { sum of }\end{array}$ \\
\hline & $\begin{array}{l}\text { Fruit and vegetables }(0-2) \text {, starchy foods }(0-1) \text {, whole } \\
\text { grain }(0-1) \text {, dairy products }(0-1) \text {, non-dairy sources } \\
\text { of animal protein }(0-1) \text {, seafood }(0-1) \text {, added fat }(0-1), \\
\text { vegetable added fat }(0-1) \text {, sweets }(-0 \cdot 5-1) \text {, water } \\
\text { and soda }(0-1) \text {, alcohol }(0-1) \text {, salt }(-0 \cdot 5-1 \cdot 5)\end{array}$ & $0-1.5$ & & 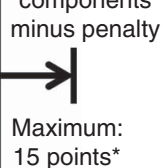 \\
\hline
\end{tabular}

PANDiet, Verger et al.

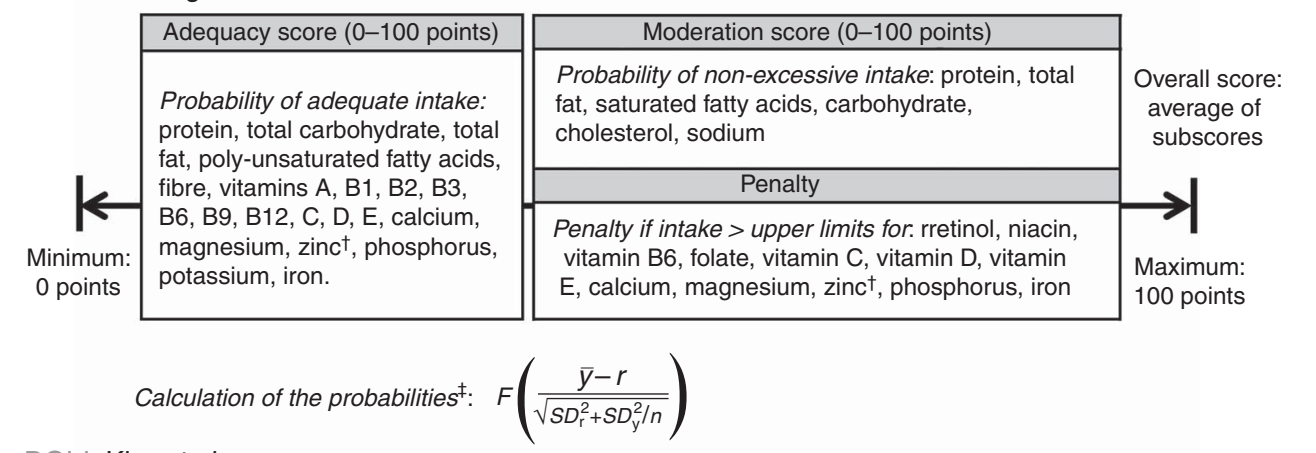

DQI-I, Kim et al.

\begin{tabular}{|l|l|l|}
\hline $\begin{array}{c}\text { Manimum:ty score } \\
\text { (points attributed) }\end{array}$ & $\begin{array}{l}\text { Odequacy score } \\
\text { (points attributed) }\end{array}$ \\
\cline { 1 - 1 } $\begin{array}{l}\text { Overall food 0-15 } \\
\text { group variety }\end{array}$ & $\begin{array}{l}\text { Adequate intake: } \\
\text { vegetables, fruits, } \\
\text { cereals, fibre, } \\
\text { protein, Fe, Ca, } \\
\text { vitamin C } \\
(0-5 \text { each) }\end{array}$ \\
\hline $\begin{array}{l}\text { Protein source } 0-5 \\
\text { variety }\end{array}$
\end{tabular}

\begin{tabular}{|c|c|c|}
\hline $\begin{array}{l}\text { Moderation } \\
\text { score (points) }\end{array}$ & $\begin{array}{l}\text { Overall balance } \\
\text { score (points) }\end{array}$ & $\begin{array}{c}\text { Overall score: } \\
\text { sum of subscores }\end{array}$ \\
\hline $\begin{array}{l}\text { Non-excessive } \\
\text { intake : total fat, } \\
\text { saturated fat, } \\
\text { cholesterol, } \mathrm{Na} \text {, } \\
\text { empty-energy } \\
\text { foods (0-6 each) }\end{array}$ & $\begin{array}{l}\text { Carbohydrate : } 0-6 \\
\text { protein : fat } \\
\text { PUFA : MUFA 0-4 } \\
\text { : SFA }\end{array}$ & $\begin{array}{l}\text { Maximum: } \\
100 \text { points }\end{array}$ \\
\hline
\end{tabular}

Fig. 1. Characteristics and computation of the investigated dietary scores. * Maximum of the modified Programme National Nutrition Santé-Guideline Score (mPNNSGS): 13.5 points. $†$ We did not have any information on $\mathrm{Zn}$ consumption, and thus did not include this variable in the calculation of the score. $\ddagger$ Probability calculation formula (PANDiet): $F$, function 'probnorm' in SAS software package; $\bar{y}$, mean intake; $r$, nutrient reference value; $s D_{r}^{2}$, interindividual variability; $s D_{y}^{2}$, day-to-day variability of intake; $n$, number of dietary record days. Probability values range from 0 to 1 , and are multiplied by 100 in order to obtain subscores of $0-100$ points. PNNS-GS, Programme National Nutrition Santé-Guideline Score; PANDiet, Probability of Adequate Nutrient Intake Dietary Score; DQI-I, Dietary Quality Index-International.

Data on treatment and storage (at $-80^{\circ} \mathrm{C}$ ) of fasting blood samples as well as the measurement of serum concentrations of vitamin C, $\beta$-carotene, $\alpha$-tocopherol, $\mathrm{Zn}, \mathrm{Se}^{(18)}$ and glucose ${ }^{(12)}$ have been described previously ${ }^{(12)}$.

\section{Events of cancer and CVD during follow-up (1994-2009)}

Data collection concerning events of cancer and CVD during follow-up has been extensively described ${ }^{(12,19)}$. In brief, an independent expert committee validated such events after review of relevant medical records, relying on the 10th International World Health Organization Classification of Diseases $^{(20)}$.

\section{Definition of 'healthy ageing'}

This study's definition of HA was largely based on the concept proposed by Rowe \& Kahn ${ }^{(2)}$. Hence, HA was defined as follows: (a) the absence of incident major chronic disease (cancer, CVD or diabetes) during follow-up, limitations in instrumental activities of daily living, function-limiting pain, depressive symptomatology and health-related limitations in social life; and (b) the presence of good physical and cognitive functioning and good overall self-perceived health (see Table 1). We defined HA as a binary variable (meeting all of the above criteria or not). An extensive description of the construction of our HA concept has been published previously ${ }^{(30)}$.

\section{Study sample selection}

We selected those participants of the SU.VI.MAX study aged 45-60 years at inclusion into the SU.VI.MAX study ( $n$ 9867), who were free of diabetes, CVD or cancer at inclusion ( $n$ 9180), and had available data for computation of the dietary scores ( $n$ 4434). After exclusion of subjects with incomplete information on HA status, a final study sample of 2329 individuals (1246 men and 1083 women) was obtained.

The explanation for the large number of individuals with missing data on HA status is that HA-related information was only collected among those SU.VI.MAX participants who decided to accept the invitation to complete the SU.VI.MAX 2 follow-up point, which was completely voluntary. Death was only a minor reason for non-participation (of the abovementioned 4434 individuals who corresponded to our inclusion criteria concerning age, prevalent diseases and dietary data, only fifty had died during follow-up). 
Table 1. Criteria used to define 'healthy ageing', SUpplémentation en Vitamines et Minéraux AntioXydants (SU.VI.MAX) and SU.VI.MAX 2 studies, France, 1994-2009*

\begin{tabular}{|c|c|c|}
\hline Criteria† & Definition & Additional information on the instrument used \\
\hline Good physical functioning & SPPB $\geq 11 / 12$ & $\begin{array}{l}\text { Physical test battery administered by trained physicians (including } \\
\text { repeated chair stands, balance testing, and gait speed testing) }\end{array}$ \\
\hline Good cognitive functioning & $\begin{array}{l}\text { MMSE } \geq 27 / 30 \text { and } \mathrm{RI}-48 \geq 19 / 48 \text { and } \\
\text { DK-TMT } \geq 5 \cdot 5\end{array}$ & $\begin{array}{l}\text { Cognitive test battery administered by trained physicians (evaluating } \\
\text { overall cognitive functioning, verbal episodic memory and executive } \\
\text { function, respectively) }\end{array}$ \\
\hline No limitations in IADL & $<1$ limitation & $\begin{array}{l}\text { Self-administered questionnaire (including, among others, questions } \\
\text { on the ability to travel, go shopping and do housekeeping) }\end{array}$ \\
\hline No depressive symptoms & CES-D $<16 / 60$ & $\begin{array}{l}\text { Self-administered questionnaire developed for the evaluation of } \\
\text { depressive symptomatology in the general population, in the context } \\
\text { of epidemiological studies }\end{array}$ \\
\hline $\begin{array}{l}\text { No health-related limitations } \\
\text { in social life }\end{array}$ & $\begin{array}{l}\text { SF-36 responses: } 1-2 \text { to item } 6 \text { and } 3-5 \text { to } \\
\text { item } 10\end{array}$ & $\begin{array}{l}\text { SF-36: very widely used, self-administered questionnaire designed to } \\
\text { measure vitality, physical functioning, bodily pain, general health } \\
\text { perceptions, physical role functioning, emotional role functioning, } \\
\text { social role functioning and mental health }\end{array}$ \\
\hline Good overall self-perceived & SF-36 responses: $1-3$ to item 1 & SF-36: see above explanations \\
\hline
\end{tabular}

health

No function-limiting pain

No incident major chronic disease

SF-36 responses: $1-3$ to item 7 and $1-2$ to item 8

No incident cancer (i.e. cancer of any kind, except for basal cell carcinoma), or CVD $\ddagger$ during follow-up

No incident diabetes during follow-up
SF-36: see above explanations

Validation of events by independent expert committee

No fasting blood glucose value $\geq 1.26 \mathrm{~g} / \mathrm{l}$, anti-diabetic medication use or self-reported diabetes at the end of follow-up

SPPB, Short Physical Performance Battery ${ }^{(21)}$; MMSE, Mini Mental State Examination ${ }^{(22,23)}$; RI-48, rappel indicé-48 items ${ }^{(24)}$; DK-TMT, Delis-Kaplan version of the Trail-making test $^{(25)}$; IADL, instrumental activities of daily living ${ }^{(26,27)}$; CES-D, Center for Epidemiologic Studies Depression Scale ${ }^{(28)}$; SF-36, Medical Outcome Study Short Form-36 ${ }^{(29)}$. * This table is based on another article in which the SU.VI.MAX definition of 'healthy ageing' was originally published ${ }^{(30)}$.

† All criteria were assessed at follow-up (2007-2009), except for events of major chronic disease, which were assessed over the follow-up (1994-2009). The test batteries were administered in visit centres within hospitals near the participants' homes, and the questionnaires were completed by the participants at home, and then verified by technicians. All subjects were free of major chronic disease at inclusion.

‡ CVD was defined as codes I20-125, 163, 165, 166, I70, 171 and 174 from the 10th International World Health Organization Classification of Diseases.

\section{Descriptive statistics}

Participant characteristics were compared across quartiles of the dietary scores, using linear contrast tests and Cochran-MantelHaenszel tests. In addition, Mann-Whitney $U$ and $\chi^{2}$ tests were used to compare participants included in our analyses with those excluded because of missing data on HA status, considering the above-mentioned sample of 4434 participants with complete dietary data as the 'source population'. Finally, we investigated the interrelations between dietary scores (using Spearman's correlations) and between components of our HA definition (using $\kappa$ coefficients).

\section{Main statistical analyses}

Logistic regression was performed to estimate the association between the modified Programme National Nutrition SantéGuideline Score (mPNNS-GS), the PANDiet and the DQI-I, modelled as quartiles, and subsequent HA. Tests for (log-)linear trend were performed by modelling the quartiles of dietary scores as ordinal variables. Model 1 was adjusted for baseline (1994-1996) age and sex, and model 2a was adjusted for age, sex, follow-up time, supplementation group, occupation, living arrangement, smoking status, educational level, follow-up time, energy intake, number of 24-h records, physical activity (except for the PNNS-GS) and alcohol intake (except for the PNNS-GS and mPNNS-GS).

As BMI may be a potential mediator of the relationship between diet and HA, we created a supplemental model, model 2b, which was further adjusted for baseline BMI. As our principal objective was to examine the overall association between diet and HA (thus including the part of this association that may be mediated by anthropometric indicators), we considered model 2a as our main model. Finally, we conducted a sensitivity analysis using versions of the mPNNS-GS from which we took out, one by one, the different components of the score. For example, one modified score was the same score as the mPNNS-GS except that the component on salt consumption was taken out, and another corresponded to the mPNNS-GS without the component on fruit and vegetable consumption. These 'reduced' scores were modelled as standardised continuous variables, and we adjusted each analysis for the component that was taken out from the score.

As there was no interaction between sex and any of the scores on HA (all $P>0 \cdot 1$ ), we analysed data from men and women simultaneously.

To partly correct for the selection bias related to exclusion of participants with missing data on HA status, all analyses were carried out using inverse probability weighting ${ }^{(31)}$. The probability of inclusion into our analysis for each individual of the 'source population' ( $n$ 4434, cf. paragraph 'Descriptive statistics') was calculated using logistic regression (as a function of baseline variables). The inverse of the probability to be included (multiplied by the sampling proportion $n_{\text {included }} / n_{\text {total }}$ ) was then used as weights for our analyses.

\section{Additional statistical analyses}

To investigate the cumulative effect of dietary quality and physical activity, we also investigated the association between 
unmodified PNNS-GS score and HA. Furthermore, in addition to models in which the scores were modelled as quartiles, we created models including the dietary scores as standardised continuous variables. In addition, as 'age' is a key notion of our study's outcome, we conducted analyses stratified by age at follow-up $(<65 v$. $\geq 65$ years).

All analyses were conducted with SAS (version 9.3; SAS Institute, Inc.). Missing values for covariables ( $n 50$ for smoking status, $n 33$ for living arrangement and $n 26$ for occupational category) were dealt with by multiple imputation. Details on how exactly multiple imputation was carried out are given in the online Supplementary Material S2.

\section{Results}

The present analysis included 2329 participants. Our study sample's mean age at follow-up was $65 \cdot 3$ (SD 4.5) years, and the mean follow-up time was 13.5 (SD 0.4 ) years. Our criteria of HA were met by $42 \%$ of men and $36 \%$ of women. The distribution of age categories at the SU.VI.MAX 2 follow-up visit according to HA status was as follows: $\mathrm{HA}=$ no: $11 \%<60$ years, $37 \%$ 60-64 years, 30\% 65-69 years, $22 \% \geq 70$ years; HA=yes: $12 \%<60$ years, $45 \% \quad 60-64$ years, 26\% 65-69 years, $18 \% \geq 70$ years.

A total of 2329 participants were included in the present analysis. Compared with them, participants who were excluded because of missing data on HA status ( $n$ 2105) were younger, less educated, more often smokers, consumed less fruits and vegetables, had lower scores on the PANDiet and the DQI-I, a higher mean BMI and a higher mean fasting blood glucose value (online Supplementary Table S3).

Tables 2 and 3 and online Supplementary Tables S4-S7 present participant characteristics according to quartiles of the dietary scores. Most of the investigated variables (sociodemographic, lifestyle, health and nutritional data) differed according to quartiles of the different dietary scores. For

Table 2. Baseline general participant characteristics according to quartiles $(Q)$ of the modified Programme National Nutrition Santé-Guideline Score (Numbers and percentages; medians and 1st, 3rd quartiles)

\begin{tabular}{|c|c|c|c|c|c|c|c|c|c|c|}
\hline \multirow[b]{2}{*}{ Baseline characteristics } & \multirow[b]{2}{*}{$n$} & \multicolumn{2}{|c|}{ Q1* } & \multicolumn{2}{|c|}{$\mathrm{Q}^{*}$} & \multicolumn{2}{|c|}{ Q3 $^{*}$} & \multicolumn{2}{|c|}{ Q4* } & \multirow[b]{2}{*}{$P \dagger$} \\
\hline & & $n$ & $\%$ & $n$ & $\%$ & $n$ & $\%$ & $n$ & $\%$ & \\
\hline Age (years) & 2329 & & & & & & & & & 0.009 \\
\hline Median & & \multicolumn{2}{|c|}{64.43} & \multicolumn{2}{|c|}{64.82} & \multicolumn{2}{|c|}{64.75} & \multicolumn{2}{|c|}{65.32} & \\
\hline 1st, 3rd quartiles & & \multicolumn{2}{|c|}{$61 \cdot 33,68 \cdot 29$} & \multicolumn{2}{|c|}{$61.63,69.08$} & \multicolumn{2}{|c|}{$61 \cdot 75,69 \cdot 17$} & \multicolumn{2}{|c|}{$61 \cdot 79,69.69$} & \\
\hline Sex & 2329 & & & & & & & & & $<0.001$ \\
\hline Men & & 402 & $66 \cdot 6$ & 306 & $57 \cdot 2$ & 302 & $47 \cdot 9$ & 236 & $42 \cdot 2$ & \\
\hline Women & & 202 & 33.4 & 229 & $42 \cdot 8$ & 329 & $52 \cdot 1$ & 323 & $57 \cdot 8$ & \\
\hline Educational level (\%) & 2329 & & & & & & & & & 0.005 \\
\hline Primary education only & & 143 & $23 \cdot 7$ & 119 & $22 \cdot 2$ & 135 & 21.4 & 93 & $16 \cdot 6$ & \\
\hline Secondary education & & 247 & 40.9 & 210 & $39 \cdot 3$ & 231 & $36 \cdot 6$ & 243 & 43.5 & \\
\hline University level & & 214 & 35.4 & 206 & 38.5 & 265 & $42 \cdot 0$ & 223 & 39.9 & \\
\hline Occupational status (\%) & 2303 & & & & & & & & & $<0.001$ \\
\hline Homemaker & & 23 & 3.9 & 41 & 7.8 & 45 & 7.2 & 64 & 11.6 & \\
\hline Manual worker & & 46 & $7 \cdot 7$ & 28 & $5 \cdot 3$ & 42 & $6 \cdot 7$ & 18 & 3.3 & \\
\hline Employees & & 335 & $56 \cdot 1$ & 285 & 53.9 & 336 & 53.8 & 304 & $55 \cdot 0$ & \\
\hline Managerial staff & & 193 & $32 \cdot 3$ & 175 & 33.1 & 201 & 32.2 & 167 & $30 \cdot 2$ & \\
\hline Living arrangement (\%) & 2296 & & & & & & & & & 0.03 \\
\hline Living alone & & 63 & $10 \cdot 6$ & 67 & $12 \cdot 7$ & 82 & 13.1 & 83 & $15 \cdot 0$ & \\
\hline Living in a couple & & 529 & 89.4 & 460 & 87.3 & 542 & 86.9 & 470 & $85 \cdot 0$ & \\
\hline Smoking habits (\%) & 2279 & & & & & & & & & $<0.001$ \\
\hline Never smoker & & 259 & 43.6 & 264 & 51.0 & 334 & $54 \cdot 0$ & 298 & $54 \cdot 3$ & \\
\hline Former smoker & & 246 & 41.4 & 200 & 38.6 & 231 & $37 \cdot 4$ & 211 & 38.4 & \\
\hline Current smoker & & 89 & $15 \cdot 0$ & 54 & 10.4 & 53 & 8.6 & 40 & $7 \cdot 3$ & \\
\hline Physical activity level (\%) & 2329 & & & & & & & & & $<0.001$ \\
\hline Irregular or none & & 369 & $61 \cdot 1$ & 301 & $56 \cdot 3$ & 327 & $51 \cdot 8$ & 272 & $48 \cdot 7$ & \\
\hline$<1 \mathrm{~h} / \mathrm{d}$ & & 122 & $20 \cdot 2$ & 121 & 22.6 & 161 & $25 \cdot 5$ & 134 & 24.0 & \\
\hline$\geq 1 \mathrm{~h} / \mathrm{d}$ & & 113 & $18 \cdot 7$ & 113 & $21 \cdot 1$ & 143 & $22 \cdot 7$ & 153 & $27 \cdot 4$ & \\
\hline $\mathrm{BMI}\left(\mathrm{kg} / \mathrm{m}^{2}\right)$ & 2329 & & & & & & & & & 0.04 \\
\hline Median & & & & & & & & & & \\
\hline 1st, 3rd quartiles & & 21.9 & $6 \cdot 48$ & 22 & $6 \cdot 20$ & $21 . \S$ & 5.88 & 21 . & 6.04 & \\
\hline Systolic blood pressure (mmHg) & 1996 & & & & & & & & & 0.04 \\
\hline Median & & & & & & & & & & \\
\hline 1st, 3rd quartiles & & 120 & $35 \cdot 0$ & 115 & $30 \cdot 0$ & 115 & $30 \cdot 0$ & 115 & $35 \cdot 0$ & \\
\hline Diastolic blood pressure $(\mathrm{mmHg})$ & 1996 & & & & & & & & & 0.003 \\
\hline Median & & & & & & & & & & \\
\hline 1st, 3rd quartiles & & & & & $5 \cdot 0$ & & & & & \\
\hline Fasting blood glucose $(\mathrm{g} / \mathrm{l})$ & 2318 & & & & & & & & & $<0.001$ \\
\hline Median & & & & & & & & & & \\
\hline 1st, 3rd quartiles & & & & & 05 & & & & & \\
\hline
\end{tabular}

* Quartile cut-off values (maximal values per quartile): Q1, 6.05; Q2, 7.25; Q3: 8.50.

† Linear contrast tests (continuous variables) or Cochran-Mantel-Haenszel tests (categorical variables). Occupational status: $\chi^{2}$-test, as the variable values were not ordinal.

$\ddagger$ Or intellectual profession. 
Table 3. Baseline nutrition-related participant characteristics according to quartiles $(\mathrm{Q})$ of the modified Programme National Nutrition Santé-Guideline Score (mPNNS-GS) (Medians and 1st, 3rd quartiles)

\begin{tabular}{|c|c|c|c|c|c|c|c|c|c|c|}
\hline \multirow[b]{2}{*}{ Baseline characteristics } & \multirow[b]{2}{*}{$n$} & \multicolumn{2}{|r|}{ Q1* } & \multicolumn{2}{|r|}{$\mathrm{Q}^{*}$} & \multicolumn{2}{|r|}{$\mathrm{Q3}^{*}$} & \multicolumn{2}{|r|}{ Q4* } & \multirow[b]{2}{*}{$P \dagger$} \\
\hline & & Median & 1st, 3rd quartiles & Median & 1st, 3rd quartiles & Median & 1st, 3rd quartiles & Median & 1st, 3rd quartiles & \\
\hline \multicolumn{11}{|l|}{ Dietary scores } \\
\hline mPNNS-GS (points) & 2329 & $5 \cdot 25$ & $4.58,5.75$ & 6.75 & $6.50,7.00$ & $7 \cdot 80$ & $7.55,8.08$ & $9 \cdot 30$ & $8.80,9.80$ & $<0.001$ \\
\hline PNNS-GS (points) & 2329 & 5.74 & $4.79,6.25$ & 7.08 & $6.73,7.80$ & $8 \cdot 30$ & $7.80,9.05$ & $10 \cdot 00$ & $9 \cdot 30,10 \cdot 75$ & $<0.001$ \\
\hline PANDiet (points) & 2329 & 60.92 & $57 \cdot 70,65 \cdot 24$ & 61.92 & $58.49,65.84$ & 63.53 & $59.59,67.40$ & 67.04 & $62.03,71.39$ & $<0.001$ \\
\hline DQI-I (points) & 2329 & $52 \cdot 24$ & $48.44,56.49$ & $54 \cdot 71$ & $51.07,58.37$ & 56.55 & $53.05,59.58$ & $60 \cdot 30$ & $56.67,64.01$ & $<0.001$ \\
\hline Alcohol consumption (g/d) & 2329 & $20 \cdot 99$ & $5 \cdot 91,36.07$ & $18 \cdot 10$ & $0.00,28 \cdot 27$ & 6.43 & $0.00,20.99$ & 5.91 & $0.00,20.99$ & $<0.001$ \\
\hline Fruit/vegetable intake $(\mathrm{g} / \mathrm{d})$ & 2329 & 337.0 & $260.5,445.0$ & 368.2 & $274.8,491.3$ & 408.4 & $322.6,509.5$ & 473.5 & $361.3,599.7$ & $<0.001$ \\
\hline Total energy intake $(\mathrm{kJ} / \mathrm{d})$ & 9744 & 10770 & 9079,12410 & 9401 & 7452,10891 & 8598 & 7109,10134 & 7912 & 6652,9376 & $<0.001$ \\
\hline Total energy intake (kcal/d) & 2329 & 2574 & 2170,2966 & 2247 & 1781,2603 & 2055 & 1699,2422 & 1891 & 1590,2241 & \\
\hline$\%$ Total fat & 2329 & 37.69 & $34.35,40.96$ & 37.69 & $34.93,40.75$ & 37.85 & $34.83,41.07$ & 37.11 & $33.43,40.41$ & 0.15 \\
\hline$\% S F A$ & 2329 & $16 \cdot 00$ & $14.31,17.62$ & $15 \cdot 63$ & $14.14,17.44$ & $15 \cdot 48$ & $13 \cdot 85,17 \cdot 10$ & 14.67 & $12 \cdot 76,16 \cdot 41$ & $<0.001$ \\
\hline$\%$ MUFA & 2329 & 13.98 & $12 \cdot 52,15 \cdot 32$ & 14.08 & $12 \cdot 92,15.59$ & 14.29 & $12 \cdot 93,15 \cdot 82$ & $14 \cdot 17$ & $12.55,15.57$ & 0.21 \\
\hline \%PUFA & 2329 & 5.06 & $4.43,5.89$ & $5 \cdot 31$ & $4.56,6.40$ & $5 \cdot 64$ & $4.87,6.69$ & $5 \cdot 86$ & $5.02,6.74$ & $<0.001$ \\
\hline \%Carbohydrates & 2329 & 38.86 & $33.94,43.64$ & $39 \cdot 27$ & $34.73,43.15$ & 39.95 & $36.15,44.08$ & 40.91 & $37.09,45 \cdot 16$ & $<0.001$ \\
\hline \%Added sugars & 2329 & $7 \cdot 77$ & $5 \cdot 46,10 \cdot 68$ & $7 \cdot 34$ & $5 \cdot 29,9.60$ & $7 \cdot 22$ & $5 \cdot 24,9 \cdot 37$ & $6 \cdot 87$ & $4.98,8.54$ & $<0.001$ \\
\hline \%Protein & 2329 & $15 \cdot 87$ & $14.55,17.46$ & 16.08 & $14 \cdot 83,17 \cdot 72$ & $16 \cdot 39$ & $15.00,18.01$ & 16.98 & $15.44,18.87$ & $<0.001$ \\
\hline \%Animal protein & 2329 & 11.57 & $9.95,13.34$ & 11.81 & $10.29,13.52$ & 11.83 & $10.32,13.72$ & $12 \cdot 38$ & $10 \cdot 48,14 \cdot 30$ & $<0.001$ \\
\hline \%Plant protein & 2329 & $4 \cdot 27$ & $3.80,4.80$ & 4.32 & $3 \cdot 88,4 \cdot 88$ & 4.51 & $4.03,5 \cdot 10$ & 4.67 & $4 \cdot 17,5 \cdot 24$ & $<0.001$ \\
\hline Dietary fibre intake (g/4184 kJ (1000 kcal)) & 2329 & 7.77 & $6.79,8.94$ & 8.44 & $7.31,9.88$ & $9 \cdot 32$ & $8 \cdot 16,10 \cdot 71$ & 10.67 & $9 \cdot 22,12 \cdot 63$ & $<0.001$ \\
\hline $\mathrm{Na}$ intake $(\mathrm{mg} / \mathrm{d})$ & 2329 & 4.07 & $3.40,4 \cdot 86$ & 3.59 & $2.88,4.40$ & 3.40 & $2 \cdot 64,4 \cdot 14$ & 2.97 & $2.42,3.58$ & $<0.001$ \\
\hline Vitamin C $(\mu \mathrm{mol} / /) \ddagger$ & 1804 & 51.73 & $36.91,63.54$ & 54.48 & $41.24,67.05$ & $55 \cdot 17$ & $44.18,67.52$ & 59.94 & $49.99,69.54$ & $<0.001$ \\
\hline Vitamin E $(\mu \mathrm{mol} / \mathrm{l}) \ddagger$ & 1991 & $30 \cdot 80$ & $26 \cdot 10,35 \cdot 30$ & $31 \cdot 20$ & $26 \cdot 80,36 \cdot 14$ & 31.00 & $26.46,36.63$ & 31.73 & $26.59,36.40$ & 0.10 \\
\hline$\beta$-Carotene $(\mu \mathrm{mol} / \mathrm{l}) \ddagger$ & 1991 & 0.44 & $0.28,0.67$ & 0.48 & $0.32,0.76$ & 0.52 & $0.34,0.83$ & 0.59 & $0.39,0.89$ & $<0.001$ \\
\hline $\mathrm{Se}(\mu \mathrm{mol} / \mathrm{l}) \ddagger$ & 2260 & $1 \cdot 10$ & $0.99,1.22$ & $1 \cdot 10$ & $0.98,1.24$ & $1 \cdot 10$ & $0.99,1.22$ & $1 \cdot 10$ & $0.99,1.23$ & 0.54 \\
\hline $\mathrm{Zn}(\mu \mathrm{mol} / \mathrm{l}) \ddagger$ & 2264 & $13 \cdot 10$ & $11.90,14.30$ & 13.35 & $12.00,14.60$ & $13 \cdot 10$ & $11.90,14.20$ & $13 \cdot 20$ & $12.00,14.40$ & 0.83 \\
\hline
\end{tabular}

PNNS-GS, Programme National Nutrition Santé-Guideline Score; PANDiet, Probability of Adequate Nutrient Intake Dietary Score; DQI-I, Dietary Quality Index-International.

* Quartile cut-offs (maximal values per quartile): Q1, 6.05; Q2, 7.25; Q3: 8.50.

† Blood serum concentrations. 
Table 4. Association between quartiles $(Q)$ of dietary scores and healthy ageing (Odds ratios and $95 \%$ confidence intervals; $n$ 2329)*

\begin{tabular}{|c|c|c|c|c|c|c|c|c|c|}
\hline & \multicolumn{2}{|c|}{ Q1 } & \multicolumn{2}{|c|}{ Q2 } & \multicolumn{2}{|c|}{ Q3 } & \multicolumn{2}{|c|}{ Q4 } & \multirow[b]{2}{*}{$P_{\text {for trend }} \dagger$} \\
\hline & OR & $95 \% \mathrm{Cl}$ & OR & $95 \% \mathrm{Cl}$ & OR & $95 \% \mathrm{Cl}$ & OR & $95 \% \mathrm{Cl}$ & \\
\hline \multicolumn{10}{|c|}{$\begin{array}{l}\text { Principal analyses } \\
\text { mPNNS-GS }\end{array}$} \\
\hline Model $1^{*}$ & 1 & - & 1.18 & $0.92,1.50$ & 1.36 & $1.08,1.72$ & 1.60 & $1.25,2.04$ & $<0.001$ \\
\hline Model 2a† & 1 & - & 1.14 & $0.88,1.46$ & 1.26 & $0.98,1.61$ & 1.44 & $1.10,1.87$ & 0.006 \\
\hline Model $2 b \ddagger$ & 1 & - & $1 \cdot 15$ & $0.89,1.48$ & 1.27 & $0.99,1.63$ & 1.45 & $1.11,1.89$ & 0.005 \\
\hline \multicolumn{10}{|l|}{ PANDiet } \\
\hline Model $1^{*}$ & 1 & - & 1.02 & $0.81,1.30$ & $1 \cdot 15$ & $0.91,1.46$ & 1.35 & $1.07,1.72$ & 0.008 \\
\hline Model 2a† & 1 & - & 1.01 & $0.79,1.28$ & $1 \cdot 15$ & $0.90,1.47$ & 1.28 & $1.00,1.64$ & 0.03 \\
\hline Model 2b‡ & 1 & - & 0.99 & $0.77,1.26$ & $1 \cdot 10$ & $0.86,1.41$ & 1.22 & $0.95,1.56$ & 0.08 \\
\hline \multicolumn{10}{|l|}{ DQI-I } \\
\hline Model $1^{*}$ & 1 & - & 1.36 & $1.07,1.73$ & 1.50 & $1.18,1.91$ & 1.39 & $1.09,1.77$ & 0.005 \\
\hline Model 2a† & 1 & - & 1.32 & $1.03,1.68$ & 1.38 & $1.08,1.77$ & 1.28 & $0.99,1.64$ & 0.05 \\
\hline Model 2b & 1 & - & 1.32 & $1.03,1.68$ & 1.39 & $1.08,1.77$ & 1.25 & $0.97,1.61$ & 0.07 \\
\hline \multicolumn{10}{|c|}{ Supplemental analyses } \\
\hline \multicolumn{10}{|l|}{ PNNS-GS } \\
\hline Model $1^{*}$ & 1 & - & 1.24 & $0.98,1.57$ & 1.41 & $1.11,1.80$ & 1.70 & $1 \cdot 33,2 \cdot 18$ & $<0.001$ \\
\hline Model 2a† & 1 & - & 1.21 & $0.95,1.55$ & 1.40 & $1.08,1.80$ & 1.64 & $1 \cdot 26,2 \cdot 13$ & $<0.001$ \\
\hline Model 2bł & 1 & - & 1.20 & $0.94,1.54$ & 1.38 & $1.07,1.79$ & 1.61 & $1 \cdot 24,2 \cdot 10$ & $<0.001$ \\
\hline
\end{tabular}

mPNNS-GS, modified Programme National Nutrition Santé-Guideline Score; PANDiet, Probability of Adequate Nutrient Intake Dietary Score; DQI-I, Dietary Quality IndexInternational; PNNS-GS, Programme National Nutrition Santé-Guideline Score.

* Model 1 is adjusted for age and sex.

† Model $2 \mathrm{a}$ is adjusted for age, sex, supplementation group, occupation, living arrangement, smoking status, educational level, follow-up time, energy intake, number of 24-h records, physical activity (except for the PNNS-GS) and alcohol intake (except for the PNNS-GS and the mPNNS-GS).

¥ Model $2 \mathrm{~b}$ is adjusted for the same variables as model $2 \mathrm{a}$, and additionally for baseline BMI. We consider model $2 \mathrm{a}$ as our main model.

instance, higher scores on the mPNNS-GS were associated with higher age, a higher probability to be female, to have a higher educational level and occupational status, a healthier lifestyle, more favourable health parameters, higher serum vitamin $C$ and $\beta$-carotene and a nutritional profile that was closer to the French official nutrition guidelines. Spearman's coefficients for the correlation of the mPNNS-GS with the PANDiet and the DQI-I were 0.33 and 0.45 , respectively (data not shown). $\kappa$ Coefficients illustrating the interrelations between the different components of our HA definition are displayed in the online Supplementary Table 88 .

Table 4 presents the association between quartiles of dietary scores and HA, providing OR with $95 \% \mathrm{CI}$ and $P$ values for linear trend. In the fully adjusted model, higher scores on the

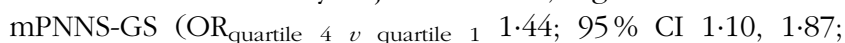
$\left.P_{\text {trend }}=0.006\right)$ and the PANDiet $(1.28 ; 95 \%$ CI $1.00,1.64$; $P_{\text {trend }}=0.03$ ), but not on the DQI-I, were related to a higher probability of HA. In fact, the highest odds for HA were not observed for quartile 4 of the DQI-I but for quartile 3 of the DQI-I - indicating the absence of a (log-)linear relationship. Our supplemental analysis concerning the PNNS-GS showed that high values on this index were linked to an even higher increase in odds of HA than high scores on the modified score mPNNS-GS.

Our models in which we additionally included baseline BMI revealed that after adjusting for BMI essentially the same results were observed for the mPNNS-GS, the observed association was slightly weaker for the DQI-I and the PNNS-GS and no longer statistically significant for the PANDiet.

Fig. 2 shows the association between standardised dietary scores (mean $=0, \mathrm{SD}=1$ ) and HA. Positive associations between the scores and HA were only observed for the mPNNS-GS and

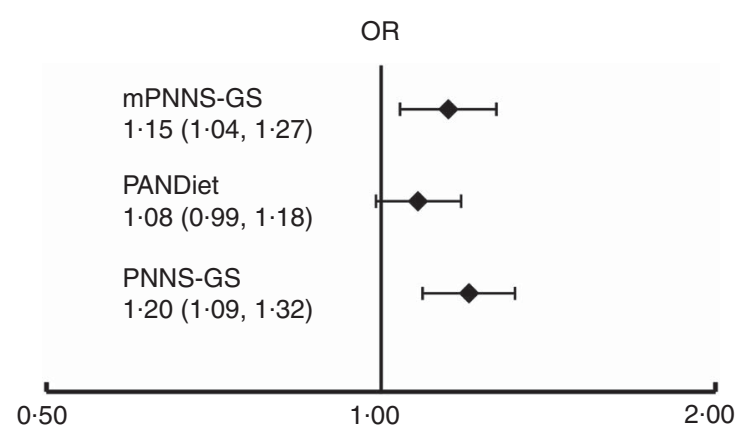

Fig. 2. Association between continuous standardised dietary scores and healthy ageing ( $n$ 2329). OR were calculated via a multivariable logistic regression model, adjusted for age, sex, supplementation group, occupation, living arrangement, smoking status, educational level, follow-up time, energy intake, number of 24-h records, physical activity (except for the Programme National Nutrition Santé-Guideline Score (PNNS-GS)) and alcohol intake (except for the PNNS-GS and the modified Programme National Nutrition Santé-Guideline Score (mPNNS-GS)). PANDiet, Probability of Adequate Nutrient Intake Dietary Score.

the PNNS-GS, but not for the PANDiet. We do not present the association between continuous DQI-I and HA as the analysis by quartiles had indicated the absence of a (log-)linear relationship.

Online Supplementary Table S9 presents analyses stratified by age at follow-up. A positive relationship between the mPNNS-GS and the probability for HA was present among both younger and older participants. Significant results for the other dietary scores were not observed in either age group. Online Supplementary Table S10 presents our analysis in which we took out the different components of the mPNNS-GS. For some of the 'reduced' scores, a slightly weakened (scores without 
seafood, without salt or without the component on meat, poultry, seafood and eggs) or a slightly strengthened (score without the component on vegetable added fats) association was observed. However, all observed OR remained quite similar to the OR observed for the mPNNS-GS.

\section{Discussion}

\section{Summary of findings}

In this large cohort of French adults, higher adherence to the French official nutrition guidelines, as measured by the mPNNS-GS, and higher adequacy regarding French nutrientbased recommendations, as measured by the PANDiet, were prospectively related to higher odds of HA.

The magnitude of these associations was stronger for the mPNNS-GS than for the PANDiet, which was only related to HA when modelled as quartiles, but not when modelled as a continuous variable. The relationship between DQI-I and HA was not linear - as the highest odds of HA were found for the third quartile. We did not attempt to model a non-linear relationship between DQI-I and HA, as nutritional indices should by definition yield the most favourable public health outcomes for the highest scores. One potential explanation for the shape of this association may be that the highest scores for the 'total fat' item of the 'moderation' subscore of the DQI-I are attributed to subjects covering $<20 \%$ of their total energy intakes by fat. Recommendations including such drastically low fat intakes have been controversially discussed in the literature ${ }^{(32,33)}$. A potential reason for the generally better performance of the mPNNS-GS in terms of predicting HA, with respect to the other tested scores, may be that only the mPNNS-GS penalises energy overconsumption. However, using an unpenalised version of the mPNNS-GS yielded very similar results as our main analyses (data not shown). The relatively low correlations between the mPNNS-GS and the PANDiet and the DQI-I indicate that the overall dietary quality is reflected in a different manner by this score than by the other scores. The main difference between the scores is that the mPNNS-GS reflects food-based dietary guidelines, whereas the PANDiet reflects nutrient-based reference recommendations, and the DQI-I reflects both food-based and nutrient-based recommendations. Moreover, only the mPNNS-GS includes a component on alcohol consumption however, our sensitivity analyses showed that a version of the mPNNS-GS in which the alcohol component was excluded yielded essentially the same results as the complete mPNNS-GS score. This was also true for the other components of the mPNNS-GS score, which did not, in an isolated manner, appear to drive the observed association of mPNNS-GS with HA. Further investigations are needed to determine to which degree adherence to food-based dietary guidelines genuinely shows a stronger association with HA than the adequacy to nutrient reference values, and to which degree such findings are influenced by technical aspects such as different types of scoring systems.

Our supplemental results concerning the association between PNNS-GS and HA suggest that the highest health benefits can be obtained by a combination of adherence to nutritional recommendations and a high physical activity level.
Moreover, in our age-stratified supplemental analyses, a positive relationship between mPNNS-GS and HA was present among both younger and older participants. The fact that we observed no significant results for the PANDiet in age-stratified analyses is probably related to limited statistical power.

Our analyses in which we additionally adjusted for baseline BMI indicate that, although the observed associations for the mPNNS-GS, the DQI-I and the PNNS-GS were probably not strongly mediated by this anthropometric indicator, there may have been a substantial mediation effect for the PANDiet. However, these results should be cautiously interpreted as we did not carry out a formal mediation analysis.

\section{Comparison with the literature}

To the best of our knowledge, only three studies have investigated the association between a priori dietary scores and HA. In a cross-sectional study based on the Nurses' Health Study ${ }^{(8)}$, higher scores on the food- and nutrient-based AHEI-2010 ${ }^{(34)}$ and on the Alternate Mediterranean diet score were associated with a higher probability of HA. In a further prospective study, there was a positive association of the adherence to the Australian food-based dietary guidelines with odds for $\mathrm{HA}^{(9)}$. In contrast, in a prospective study based on the Whitehall II cohort ${ }^{(6)}$, lower adherence to the original AHEI was unrelated to HA.

In the study published by Akbaraly et al. ${ }^{(6)}$, the relationship between a posteriori dietary patterns and HA was also investigated, and an inverse association between a Western-type dietary pattern and the probability of HA was observed. This is in line with a study based on the Melbourne Collaborative Cohort $^{(7)}$, which observed an inverse association between a pattern characterised by high consumption of meat and fatty foods and HA, in addition to a positive association between a high fruit dietary pattern and HA.

In a previous investigation of data from the SU.VI.MAX study $^{(35)}$, no relationship between a Western-type pattern and HA was observed. On the other hand, there was a positive prospective association between a bealthy-type dietary pattern and HA among participants with below-median energy intakes, underlying the importance of both high dietary quality and regulated energy intake.

One aspect that should be taken into consideration when comparing the present study with the above-mentioned other studies are substantial differences in the applied HA concepts. The criteria applied by Akbaraly et al. (surviving to $\geq 60$ years, absence of chronic conditions and mental health problems, presenting above sex- and age-specific median performance in cardiometabolic, respiratory, musculoskeletal and cognitive functioning tests) and Samieri et al. (survival to $\geq 70$ years, absence of major chronic disease and of major impairments in cognitive, physical and mental functioning) appear as particularly severe, as only 4 and $11 \%$ of participants were identified as 'ideal' or 'healthy' agers, respectively. Hodge et al. (HA definition: surviving to at least age 70 years, good mental health, absence of major chronic disease and of major limitations in physical functioning) and Gopinath et al. (HA definition: absence of disability, depressive symptoms, cognitive impairment, respiratory symptoms and chronic diseases) identified 19 
and $16 \%$ of participants as 'successful' agers, respectively. In our study, even more individuals (39\%) were classed has 'healthy' agers. This is probably related to the fact that, in contrast to the above-cited studies, survival to a specific age was not part of our HA definition. However, differences in HA proportions across studies are not merely a function of age but also of the chosen indicators and cut-off values, as is illustrated by the fact that the mean age at HA assessment was about 67 years in the study by Akbaraly et al. (showing very low HA proportions) and about 76 years in the study by Hodge et al. (showing markedly higher HA proportions).

In contrast to the limited literature on the link between overall diet and HA, the number of publications on the association between holistic representations of the diet and specific diseases has much increased during the past few dec$\operatorname{ades}^{(3,5,36-39)}$. A review article concluded that observational studies suggested a modest favourable role of 'healthful' dietary patterns concerning all-cause mortality and CHD - but did not consistently suggest a role concerning cancer ${ }^{(5)}$. Moreover, a recent publication issued from a conference on 'Nutrition and healthy ageing' concluded that the available studies globally suggested an important role of 'dietary patterns rich in fruit, vegetables, fish, whole grains and starchy low-fat staple foods' for HA-related outcomes such as life expectancy, cardiometabolic health and cognitive health ${ }^{(3)}$

Overall, scientific literature on the link between overall diet and health status including physical and cognitive functioning among elderly persons is scant, and does not yet permit an estimation of the quantity of a potential protective effect of a high-quality overall diet.

\section{Limitations and strengths}

Some limitations of our study should be noted. First, published definitions of HA are quite heterogeneous ${ }^{(1)}$. However, the HA model used in the present study was largely based on the 'classical' concept proposed by Rowe \& Kahn ${ }^{(2)}$. Second, in our study, HA status was not available at baseline (1994-1996). Yet, subjects in our study were only aged 45-60 years at inclusion into the SU.VI.MAX trial and free of major chronic disease. Accordingly, our working hypothesis that individuals were initially healthy is quite plausible. Third, the external validity of our results may be limited as the SU.VI.MAX participants are a sample of selected volunteers. Notably, individuals with a particularly poor overall diet may be under-represented in a nutrition-related study. Although potential selection bias was addressed by inverse probability weighting, this may have been insufficient to fully counterbalance the observed differences between excluded and included participants concerning important lifestyle and health characteristics. Finally, given our observational study design, we cannot exclude potential residual confounding. Important strengths of our analysis are the prospective study design, the use of a large set of HA indicators and the availability of accurate nutritional data, given the availability of, on average, ten dietary records per subject.

In conclusion, this study suggests a beneficial role of high adherence to the food-based and nutrient-based French nutritional recommendations for a HA process, including the avoidance of chronic disease, good physical and cognitive functioning, as well as good self-perceived, mental and social health. The indicator that was most strongly related to a higher probability of HA in our study was the PNNS-GS, which reflects both the food-based items and the physical activity item of the French official nutrition guidelines. This suggests a high pertinence of the French official nutrition guidelines for the prevention of age-related health decline, and more generally a high importance of both high dietary quality and adequate physical activity level for health ageing. Further prospective observational and intervention studies are needed to confirm these findings.

\section{Acknowledgements}

The authors thank Younes Esseddik, Gwenaël Monot, Paul Flanzy, Mohand Ait Oufella, Yasmina Chelghoum and Than Duong Van (computer scientists), Rachida Mehroug (logistic assistant) and Nathalie Arnault, Véronique Gourlet, Fabien Szabo, Laurent Bourhis and Stephen Besseau (statisticians) for their technical contribution to the SU.VI.MAX study. The authors also thank Stéphane Raffard, who was responsible for standardisation of the cognitive evaluation, and Frédérique Ferrat, who coordinated the logistic aspects of the neuropsychological evaluation.

This study was funded by the French National Research Agency (no. ANR-05-PNRA-010), the French Ministry of Health, Médéric, Sodexo, Ipsen, Mutuelle générale de l'Éducation nationale (MGEN) and Pierre Fabre. Mederic and MGEN are French health insurance organisations, which are complementary to the National Health Insurance System. Ipsen and Pierre Fabre are private pharmaceutical companies. Sodexo, a food catering company, supported the study by organising meetings between researchers and study participants. The funding bodies did not have any involvement in the design/ conduct of the research, in data analysis/interpretation or in writing/approval of the manuscript. K. E. A. was supported by a doctoral fellowship from the Ecole Doctorale Galilée, University of Paris 13, Sorbonne Paris Cité. None of the funders had any role in study design, data collection and analysis, decision to publish or writing of the manuscript.

S. H., P. G. and E. K.-G. designed the study (development of the concept, design and protocol of the SU.VI.MAX/ SU.VI.MAX 2 studies and coordinating of data collection); K. E. A. performed the statistical analysis and wrote the article; E. K.-G. provided methodological guidance; K. E. A., V. A. A., G. M. C., E. O. V., C. J., S. H., P. G. and E. K.-G. were involved in interpreting the results and editing the manuscript for important intellectual content; E. K.-G. and K. E. A. had primary responsibility for the final content. All the authors read and approved the final manuscript.

E. O. V. was supported by Danone Research and a grant from the Association nationale de la recherche et de la technologie (CIFRE 474/2010) from October 2010 to November 2013. Danone Research had no role in study design, data collection and analysis, decision to publish or preparation of the manuscript. None of the other authors declare any conflicts of interest. 


\section{Supplementary material}

For supplementary material/s referred to in this article, please visit http://dx.doi.org/doi:10.1017/S0007114516002233

\section{References}

1. Cosco TD, Prina AM, Perales J, et al. (2014) Operational definitions of successful aging: a systematic review. Int Psychogeriatr 26, 373-381.

2. Rowe JW \& Kahn RL (1997) Successful aging. Gerontologist 37, 433-440.

3. Kiefte-de Jong JC, Mathers JC \& Franco OH (2014) Nutrition and healthy ageing: the key ingredients. Proc Nutr Soc 73, 249-259.

4. Estaquio C, Kesse-Guyot E, Deschamps V, et al. (2009) Adherence to the French Programme National Nutrition Sante Guideline Score is associated with better nutrient intake and nutritional status. J Am Diet Assoc 109, 1031-1041.

5. Kant AK (2010) Dietary patterns: biomarkers and chronic disease risk. Appl Physiol Nutr Metab 35, 199-206.

6. Akbaraly T, Sabia S, Hagger-Johnson G, et al. (2013) Does overall diet in midlife predict future aging phenotypes? A cohort study. Am J Med 126, 411-419.

7. Hodge AM, O'Dea K, English DR, et al. (2014) Dietary patterns as predictors of successful ageing. J Nutr Health Aging 18, 221-227.

8. Samieri C, Sun Q, Townsend MK, et al. (2013) The association between dietary patterns at midlife and health in aging: an observational study. Ann Intern Med 159, 584-591.

9. Gopinath B, Russell J, Kifley A, et al. (2016) Adherence to dietary guidelines and successful aging over 10 years. J Gerontol A Biol Sci Med Sci 71, 349-355.

10. Verger EO, Mariotti F, Holmes BA, et al. (2012) Evaluation of a diet quality index based on the probability of adequate nutrient intake (PANDiet) using national French and US dietary surveys. PLOS ONE 7, e42155.

11. Kim S, Haines PS, Siega-Riz AM, et al. (2003) The Diet Quality Index-International (DQI-I) provides an effective tool for cross-national comparison of diet quality as illustrated by China and the United States. J Nutr 133, 3476-3484.

12. Hercberg S, Galan P, Preziosi P, et al. (2004) The SU.VI.MAX Study: a randomized, placebo-controlled trial of the health effects of antioxidant vitamins and minerals. Arch Intern Med 164, 2335-2342.

13. Hercberg S, Galan P, Preziosi P, et al. (1998) Background and rationale behind the SU.VI.MAX Study, a prevention trial using nutritional doses of a combination of antioxidant vitamins and minerals to reduce cardiovascular diseases and cancers. SUpplementation en VItamines et Mineraux AntioXydants Study. Int J Vitam Nutr Res 68, 3-20.

14. Kesse-Guyot E, Amieva H, Castetbon K, et al. (2011) Adherence to nutritional recommendations and subsequent cognitive performance: findings from the prospective Supplementation with Antioxidant Vitamins and Minerals 2 (SU.VI.MAX 2) study. Am J Clin Nutr 93, 200-210.

15. Le Moullec N, Deheeger M, Preziosi P, et al. (1996) Validation du manuel photos utilisé pour l'enquête alimentaire de l'étude SU.VI.MAX (Validation of the photography-based instruction manual used for the dietary assessment of the SU.VI.MAX study). Cah Nutr Diet 31, 158-164.

16. Kesse-Guyot E, Touvier M, Henegar A, et al. (2011) Higher adherence to French dietary guidelines and chronic diseases in the prospective SU.VI.MAX cohort. Eur J Clin Nutr 65, 887-894.
17. Lasfargues G, Vol S, Le CH, et al. (1990) Validity of a short selfadministered dietary questionnaire compared with a dietetic interview. Presse Med 19, 953-957 (in French).

18. Kesse-Guyot E, Fezeu L, Jeandel C, et al. (2011) French adults' cognitive performance after daily supplementation with antioxidant vitamins and minerals at nutritional doses: a post hoc analysis of the Supplementation in Vitamins and Mineral Antioxidants (SU.VI.MAX) trial. Am J Clin Nutr 94, 892-899.

19. Hercberg S, Kesse-Guyot E, Druesne-Pecollo N, et al. (2010) Incidence of cancers, ischemic cardiovascular diseases and mortality during 5-year follow-up after stopping antioxidant vitamins and minerals supplements: a postintervention followup in the SU.VI.MAX Study. Int J Cancer 127, 1875-1881.

20. World Health Organization (2003) International Classification of Diseases, 10th Revision Clinical Modification. Geneva: WHO.

21. Guralnik JM, Simonsick EM, Ferrucci L, et al. (1994) A short physical performance battery assessing lower extremity function: association with self-reported disability and prediction of mortality and nursing home admission. J Gerontol 49 , M85-M94

22. Folstein MF, Robins LN \& Helzer JE (1983) The mini-mental state examination. Arch Gen Psychiatry 40, 812.

23. Pendlebury ST, Cuthbertson FC, Welch SJ, et al. (2010) Underestimation of cognitive impairment by Mini-Mental State Examination versus the Montreal Cognitive Assessment in patients with transient ischemic attack and stroke: a population-based study. Stroke 41, 1290-1293.

24. Adam S, Van der LM, Ivanoiu A, et al. (2007) Optimization of encoding specificity for the diagnosis of early $\mathrm{AD}$ : the RI-48 task. J Clin Exp Neuropsychol 29, 477-487.

25. Delis DC, Kaplan E \& Kramer JH (2001) Delis-Kaplan Executive Function System (D-KEFS) Examiner's Manual, 1st ed. San Antonio, TX: The Psychological Corporation.

26. Graf C (2008) The Lawton instrumental activities of daily living (IADL) scale. Medsurg Nurs 17, 343-344.

27. Delis DC, Kaplan E \& Kramer JH (2001) Delis-Kaplan Executive Function System (D-KEFS) Technical Manual, 1st ed. San Antonio, TX: The Psychological Corporation.

28. Morin AJ, Moullec G, Maiano C, et al. (2011) Psychometric properties of the Center for Epidemiologic Studies Depression Scale (CES-D) in French clinical and nonclinical adults. Rev Epidemiol Sante Publique 59, 327-340.

29. Leplege A, Ecosse E, Verdier A, et al. (1998) The French SF-36 Health Survey: translation, cultural adaptation and preliminary psychometric evaluation. J Clin Epidemiol 51, 1013-1023.

30. Assmann KE, Andreeva VA, Jeandel C, et al. (2015) Healthy aging five years after a period of daily supplementation with antioxidant nutrients: a post hoc analysis of the French randomised trial SU.VI.MAX. Am J Epidemiol 182, 694-704.

31. Seaman SR \& White IR (2011) Review of inverse probability weighting for dealing with missing data. Stat Methods Med Res 22, 278-295.

32. Sanders TA (2003) High- versus low-fat diets in human diseases. Curr Opin Clin Nutr Metab Care 6, 151-155.

33. Cheung ST (2000) Possible dangers in a low fat diet: some evidence reviewed. Nutr Health 14, 171-280.

34. McCullough ML, Feskanich D, Stampfer MJ, et al. (2002) Diet quality and major chronic disease risk in men and women: moving toward improved dietary guidance. Am J Clin Nutr 76, 1261-1271.

35. Assmann KE, Lassale C, Andreeva VA, et al. (2015) A healthy dietary pattern at midlife, combined with a regulated energy intake, is related to increased odds for healthy aging. $J$ Nutr 145, 2139-2145. 
36. Waijers PM, Feskens EJ \& Ocke MC (2007) A critical review of predefined diet quality scores. Br J Nutr 97, 219-231.

37. Sofi F, Abbate R, Gensini GF, et al. (2010) Accruing evidence on benefits of adherence to the Mediterranean diet on health: an updated systematic review and meta-analysis. Am J Clin Nutr 92, 1189-1196.
38. Kourlaba G \& Panagiotakos DB (2009) Dietary quality indices and human health: a review. Maturitas 62, 1-8.

39. Kontogianni MD \& Panagiotakos DB (2014) Dietary patterns and stroke: a systematic review and re-meta-analysis. Maturitas 79, 41-47. 